\title{
Circulating oxidised low-density lipoprotein and intercellular adhesion molecule- 1 and risk of type 2 diabetes mellitus: the Atherosclerosis Risk in Communities Study
}

\author{
R. C. Hoogeveen - C. M. Ballantyne $\cdot$ H. Bang • \\ G. Heiss • B. B. Duncan • A. R. Folsom • J. S. Pankow
}

Received: 11 August 2006 / Accepted: 13 October 2006 / Published online: 29 November 2006

(C) Springer-Verlag 2006

\begin{abstract}
Aims/hypothesis To evaluate the role of oxidative stress and inflammation in the aetiology of type 2 diabetes, we examined the association of oxidised LDL (ox-LDL) and soluble intercellular adhesion molecule-1 (sICAM-1) levels with type 2 diabetes incidence over 9 years in the Atherosclerosis Risk in Communities Study.

Materials and methods In a large, prospective, case-cohort design, ox-LDL and sICAM-1 were measured in stored plasma samples collected at baseline in stratified samples of 581 diabetes cases and 572 non-cases selected from 10,275
\end{abstract}

R. C. Hoogeveen $(\bowtie) \cdot$ C. M. Ballantyne

Section of Atherosclerosis and Vascular Medicine,

Department of Medicine, Baylor College of Medicine, and Center for Cardiovascular Disease Prevention,

Methodist DeBakey Heart Center,

Houston, TX, USA

e-mail: ronh@bcm.tmc.edu

H. Bang

Division of Biostatistics and Epidemiology,

Department of Public Health,

Weill Medical College of Cornell University,

New York, NY, USA

G. Heiss $\cdot$ B. B. Duncan

Department of Epidemiology, School of Public Health, University of North Carolina,

Chapel Hill, NC, USA

B. B. Duncan

Graduate Studies Program in Epidemiology,

School of Medicine, Federal University of Rio Grande do Sul,

Porto Alegre, RS, Brazil

\section{A. R. Folsom · J. S. Pankow}

Division of Epidemiology and Community Health,

School of Public Health, University of Minnesota,

Minneapolis, MN, USA middle-aged men and women without prevalent diabetes at baseline.

Results Compared with non-cases, diabetes cases had significantly higher mean baseline levels of ox-LDL and sICAM-1. Elevated ox-LDL and sICAM-1 were both associated with increased risk of incident diabetes after adjustment for age, sex, race and centre, with hazard ratios for the highest vs lowest tertiles of 1.68 (95\% CI $1.25-$ 2.24 ) and 1.91 (95\% CI 1.45-2.50), respectively. After additional adjustment for fasting glucose, waist circumference, HDL-cholesterol, triacylglycerol, hypertension and C-reactive protein, only sICAM-1 remained an independent predictor of incident diabetes (hazard ratio 1.50; 95\% CI 1.02-2.23).

Conclusions/interpretation In this community-based cohort of middle-aged US adults, elevated plasma ox-LDL and sICAM-1 levels were associated with increased risk of type 2 diabetes. Measurement of ICAM-1 or ox-LDL, or other measures related to inflammation or oxidative stress, may be helpful in identifying those patient populations in which to test whether novel therapies that inhibit specific pathways related to inflammation or oxidative stress are beneficial in the prevention of diabetes in humans.

Keywords Diabetes $\cdot$ ICAM- 1 Inflammation .

Oxidised LDL

\begin{tabular}{|c|c|}
\hline \multicolumn{2}{|c|}{ Abbreviations } \\
\hline AIR Study & $\begin{array}{l}\text { Atherosclerosis and Insulin Resistance } \\
\text { Study }\end{array}$ \\
\hline ARIC Study & $\begin{array}{l}\text { Atherosclerosis Risk in Communities } \\
\text { Study }\end{array}$ \\
\hline CRP & C-reactive protein \\
\hline HDL-C & HDL-cholesterol \\
\hline HR & hazard ratio \\
\hline
\end{tabular}


ICAM-1 intercellular adhesion molecule-1

LDL-C LDL-cholesterol

ox-LDL oxidised LDL

sICAM-1 soluble intercellular adhesion molecule-1 representative measures of oxidative stress and low-grade inflammation, with the risk of development of type 2 diabetes in middle-aged men and women in the large, prospective, bi-racial Atherosclerosis Risk in Communities (ARIC) Study.

\section{Subjects and methods}

Study population In 1987-1989, the ARIC Study recruited a population-based cohort of 15,792 men and women aged 45-64 years from four US communities [23]. Follow-up examinations were conducted at approximately 3-year intervals and included assessment for incident diabetes. Human subjects research review committees at the involved institutions approved the study, and all participants gave written consent. We chose the same case-cohort design previously used to investigate the role of inflammation in the development of diabetes [12]. After exclusions for preexisting diabetes or insufficient data, 10,275 subjects remained, of whom 1,155 had incident diabetes. Subjects were stratified by race (African American or white). A random sample of cases $(n=581)$ and of the cohort $(n=693$, including 76 with incident diabetes) were selected within each racial group. Exclusion of subjects with non-fasting samples or missing covariate values left 581 cases and 572 non-cases for analysis. Sampling fractions were 72 and $39 \%$ of African American and white cases and 15 and 5\% of African American and white members of the eligible cohort, respectively.

Clinical and laboratory measurements We analysed oxLDL and soluble ICAM-1 (sICAM-1) levels at a central laboratory in plasma collected at the baseline examination and stored for 15 years at $-70^{\circ} \mathrm{C}$. ox-LDL levels were measured in duplicate by a sandwich ELISA using monoclonal capture antibody $\mathrm{mAb}^{-4 E 6^{6}}$ (Mercodia AB, Uppsala, Sweden) and averaged. sICAM-1 levels were measured in duplicate by sandwich ELISA (R\&D Systems Inc., Minneapolis, MN, USA) according to the manufacturer's protocol and averaged. Reliability coefficients for ox-LDL and sICAM-1 were 0.67 and 0.98 , respectively, based on blinded replicate pairs of samples of 36 participants at the baseline examination. Interassay $\mathrm{CV}$ values for ox-LDL and sICAM-1 were 10.5 and 5.0\%, respectively.

Definitions and methods used for other baseline measures have been previously reported [12, 24]. Information on multiple markers of systemic inflammation was integrated into an inflammation score, which ranged from 0 to 6 and attributed 1 point for a value greater than the median of the cohort sample for each of IL-6, C-reactive protein (CRP), orosomucoid, sialic acid, white cell count and
The objective of the present study was to investigate the association of plasma levels of ox-LDL and ICAM-1, as 
fibrinogen [12]. Metabolic syndrome was assessed by the criteria of the National Cholesterol Education Program Adult Treatment Panel III [25].

Diagnosis of diabetes Diabetes was defined by reported physician diagnosis, use of antidiabetes medications, fasting $(\geq 8 \mathrm{~h})$ serum glucose $\geq 7.0 \mathrm{mmol} / \mathrm{l}(126 \mathrm{mg} / \mathrm{dl})$ or nonfasting glucose $\geq 11.1 \mathrm{mmol} / \mathrm{l}(200 \mathrm{mg} / \mathrm{dl})$. Date of diabetes incidence was estimated by linear interpolation using glucose values at the ascertaining examination and the previous one, as previously described [12].

Statistical analysis Statistical analysis was based on our case-cohort sampling design. We used weighted analysis of covariance to compute adjusted means and proportions of sociodemographic variables and risk factors, and Spearman correlations to describe unadjusted associations between study variables. Weights were defined as the inverse of the ethnicity-specific sampling fractions, permitting statistical estimation and inference relevant to the entire cohort. The adjusted relative risk of developing diabetes at different levels of ox-LDL and sICAM-1 was estimated by weighted proportional hazards regression as implemented in SUDAAN [26]. We used the Wald test of interaction terms in these models to test heterogeneity in associations. A test for interaction was performed in fully adjusted models using continuous covariates of SICAM and ox-LDL and one interaction term. Analyses were performed using SAS [27] and SUDAAN [28].

\section{Results}

Baseline characteristics of the study population are shown in Table 1. Compared with non-cases, individuals who developed diabetes had significantly higher mean values for BMI, fasting glucose, insulin, total cholesterol, LDLcholesterol (LDL-C) and triacylglycerol and significantly lower HDL-cholesterol (HDL-C) levels. Hypertension and parental history of diabetes were more prevalent among incident diabetes cases. Weighted mean levels of ox-LDL and sICAM-1 were significantly higher in cases than in non-cases (42.6 vs $39.3 \mathrm{U} / 1$ and 288.6 vs $265.2 \mathrm{ng} / \mathrm{ml}$, respectively; both with $p<0.001)$.

Plasma sICAM-1 level was positively correlated with circulating levels of the inflammatory markers used to determine inflammation score, with Spearman correlation coefficients ranging from 0.09 to 0.23 (Table 2). With the exception of a modest positive correlation with sialic acid $(R=0.12)$, no significant correlations were present between plasma levels of ox-LDL and the other inflammatory markers. When we examined correlations with elements
Table 1 Weighted adjusted means or prevalences of risk factors at baseline in relation to diabetes incidence

\begin{tabular}{|c|c|c|c|}
\hline Variable & $\begin{array}{l}\text { Incident diabetes } \\
(n=581)\end{array}$ & $\begin{array}{l}\text { Non-cases } \\
(n=572)\end{array}$ & $p$ value \\
\hline Age (years) ${ }^{\mathrm{a}}$ & 53.5 & 52.7 & - \\
\hline Male $(\%)^{\mathrm{a}}$ & 42.6 & 36.4 & - \\
\hline African American $(\%)^{\mathrm{a}}$ & 33.7 & 19.3 & - \\
\hline Current smoker (\%) & 19.8 & 21.9 & 0.4 \\
\hline $\begin{array}{l}\text { Parental history of } \\
\text { diabetes }(\%)\end{array}$ & 35.1 & 20.5 & $<0.001$ \\
\hline $\begin{array}{l}\text { Fasting glucose } \\
(\mathrm{mmol} / \mathrm{l})\end{array}$ & 6.0 & 5.4 & $<0.001$ \\
\hline $\begin{array}{l}\text { Fasting insulin } \\
(\mathrm{pmol} / \mathrm{l})\end{array}$ & 116.2 & 66.4 & $<0.001$ \\
\hline BMI $\left(\mathrm{kg} / \mathrm{m}^{2}\right)$ & 30.5 & 26.8 & $<0.001$ \\
\hline Hypertension (\%) & 40.5 & 23.6 & $<0.001$ \\
\hline $\begin{array}{l}\text { Total cholesterol } \\
(\mathrm{mmol} / \mathrm{l})\end{array}$ & 5.53 & 5.41 & 0.079 \\
\hline $\begin{array}{l}\text { Triacylglycerol } \\
(\mathrm{mmol} / \mathrm{l})\end{array}$ & 1.74 & 1.33 & $<0.001$ \\
\hline HDL-C (mmol/l) & 1.20 & 1.40 & $<0.001$ \\
\hline LDL-C (mmol/1) & 3.55 & 3.42 & 0.029 \\
\hline ox-LDL (U/1) & 42.6 & 39.3 & $<0.001$ \\
\hline ICAM-1 (ng/ml) & 288.6 & 265.2 & $<0.001$ \\
\hline Inflammation score & 3.91 & 2.84 & $<0.001$ \\
\hline
\end{tabular}

${ }^{a}$ Unadjusted mean or proportion; all others were adjusted for age, race, sex and centre

of the metabolic syndrome, we found that the plasma oxLDL level was positively correlated with triacylglycerol, waist circumference, blood glucose and systolic BP (Spearman correlation coefficients ranging from 0.04 to

Table 2 Correlation of ox-LDL and ICAM-1 with elements of the metabolic syndrome and inflammation mediators in the ARIC Study cohort random sample

\begin{tabular}{|c|c|c|c|c|}
\hline \multirow[t]{2}{*}{ Factor } & \multicolumn{2}{|l|}{ ox-LDL } & \multicolumn{2}{|l|}{ ICAM-1 } \\
\hline & $\begin{array}{l}\text { Spearman } \\
R\end{array}$ & $\begin{array}{l}p \\
\text { value }\end{array}$ & $\begin{array}{l}\text { Spearman } \\
R\end{array}$ & $\begin{array}{l}p \\
\text { value }\end{array}$ \\
\hline \multicolumn{5}{|l|}{ Inflammatory markers } \\
\hline Orosomucoid & 0.02 & 0.69 & 0.23 & $<0.001$ \\
\hline CRP & 0.04 & 0.27 & 0.12 & 0.001 \\
\hline IL-6 & -0.02 & 0.59 & 0.19 & $<0.001$ \\
\hline $\begin{array}{l}\text { White blood cell } \\
\text { count }\end{array}$ & -0.02 & 0.53 & 0.21 & $<0.001$ \\
\hline Sialic acid & 0.12 & 0.002 & 0.10 & 0.013 \\
\hline Fibrinogen & 0.004 & 0.91 & 0.09 & 0.021 \\
\hline Inflammation score & 0.03 & 0.40 & 0.22 & $<0.001$ \\
\hline \multicolumn{5}{|c|}{ Metabolic syndrome elements } \\
\hline HDL-cholesterol & -0.25 & $<0.001$ & -0.18 & $<0.001$ \\
\hline Triacylglycerol & 0.28 & $<0.001$ & 0.20 & $<0.001$ \\
\hline Waist circumference & 0.13 & 0.001 & 0.06 & 0.15 \\
\hline Fasting glucose & 0.10 & 0.01 & -0.01 & 0.88 \\
\hline Systolic BP & 0.04 & 0.29 & -0.01 & 0.86 \\
\hline
\end{tabular}


$0.28)$ and negatively correlated with HDL-C $(R=-0.25)$. Not surprisingly, plasma ox-LDL levels had a strong positive correlation with LDL-C $(R=0.56, p<0.0001)$. Circulating sICAM-1 levels were positively correlated with plasma triacylglycerol $(R=0.20)$ and negatively correlated with plasma HDL-C $(R=-0.18)$. No significant correlations were found between plasma sICAM-1 levels and waist circumference, fasting glucose or systolic BP.

Adjusted mean baseline values of ox-LDL and sICAM-1 in different subgroups of the cohort random sample are presented in Table 3. Plasma ox-LDL levels were significantly higher in men than in women (41.6 vs $38.0 \mathrm{U} / \mathrm{l}$, $p=0.005)$ and in individuals with the metabolic syndrome vs those without the metabolic syndrome (42.3 vs $38.7 \mathrm{U} / \mathrm{l}$, $p=0.031$ ). No significant differences in plasma ox-LDL levels were found between whites and African Americans, current smokers and non-smokers, or individuals with or without systemic inflammation as determined by inflammatory score. Mean plasma sICAM-1 levels were significantly higher in whites than in African Americans (278.0 vs $218.1 \mathrm{ng} / \mathrm{ml}, p<0.0001)$, current smokers vs non-smokers (317.0 vs $251.4 \mathrm{ng} / \mathrm{ml}, p<0.001)$, and individuals with a higher inflammation score vs those with a low inflammation score ( 284.7 vs $254.8 \mathrm{ng} / \mathrm{ml}, p<0.001$ ). No significant differences in plasma sICAM-1 levels were found between men and women or between individuals with the metabolic syndrome and those without the metabolic syndrome.

Table 4 shows the results of proportional hazards regression analyses associating incident diabetes with baseline ox-LDL and sICAM-1 levels modelled in tertiles. The absolute risk of incident diabetes over 9 years was $11.2 \%$ (i.e. $1.2 \%$ per year). ox-LDL had a significant positive association with incident diabetes, with individuals with ox-LDL levels in the highest tertile having a $68 \%$ higher risk of developing diabetes (hazard ratio [HR] 1.68 ; $95 \%$ CI $1.25-2.24$ ) in the basic model adjusted for age, sex, race and centre (model 1). After additional adjustment for fasting glucose (model 2), waist circumference, HDL-C, triacylglycerol and hypertension (model 3), and CRP (model 4), this association was attenuated and not statistically significant. Circulating sICAM-1 levels were also positively associated with incident diabetes. In model 1 , individuals with SICAM-1 values in the highest tertile had a 91\% higher risk of developing diabetes (HR 1.91; 95\% CI 1.45-2.50). HRs were slightly attenuated in further adjusted models, but sICAM-1 remained an independent predictor in a model adjusted for age, sex, race, centre, fasting glucose, waist circumference, HDL-C, triacylglycerol, hypertension and CRP (HR 1.50; 95\% CI 1.02-2.23). A statistically significant interaction between ox-LDL and sICAM-1 on risk of developing diabetes was found in all the models tested (e.g. $p=0.01$ in the fully adjusted model). Results of proportional hazards regression analyses that jointly accounted for ox-LDL and sICAM-1 are summarised in Fig. 1. Individuals in the highest tertile for both ox-LDL and sICAM-1 were at the highest risk of developing diabetes (HR 2.43; 95\% CI 1.43-4.13 [model 2] and HR 1.94; 95\% CI 1.03-3.64 [model 4] compared with individuals in the lowest tertiles for both). Individuals with either high ox-LDL and low-to-medium sICAM-1 or high sICAM-1 and low-tomedium ox-LDL did not have a significantly increased risk of developing diabetes compared with those in the lowest tertiles for both.
Table 3 Adjusted $^{\mathrm{a}}$ means of ox-LDL and ICAM-1 in subgroups of the cohort random sample

\footnotetext{
${ }^{a}$ Adjusted through a weighted analysis of covariance for age, sex, ethnicity and smoking status
}

\begin{tabular}{|c|c|c|c|c|c|c|}
\hline \multirow[t]{2}{*}{ Group } & \multicolumn{3}{|c|}{ ox-LDL (U/l) } & \multicolumn{3}{|c|}{ ICAM-1 (ng/ml) } \\
\hline & Mean & $95 \% \mathrm{CI}$ & $p$ & Mean & $95 \% \mathrm{CI}$ & $p$ \\
\hline Overall & 39.3 & $38.1-40.5$ & & 266.0 & $259.5-272.5$ & \\
\hline Sex & & & 0.005 & & & 0.448 \\
\hline Men & 41.6 & $39.6-43.6$ & & 263.0 & $254.3-271.7$ & \\
\hline Women & 38.0 & $36.5-39.5$ & & 267.7 & $258.9-276.5$ & \\
\hline Ethnicity & & & 0.595 & & & $<0.001$ \\
\hline White & 39.4 & $38.0-40.9$ & & 278.0 & $270.6-285.5$ & \\
\hline African American & 38.8 & $37.1-40.5$ & & 218.1 & $205.1-231.2$ & \\
\hline Current smoker & & & 0.321 & & & $<0.001$ \\
\hline Yes & 40.5 & $37.8-43.1$ & & 317.0 & $300.9-333.1$ & \\
\hline No & 39.0 & $37.7-40.3$ & & 251.4 & $244.1-258.6$ & \\
\hline Metabolic syndrome & & & 0.031 & & & 0.287 \\
\hline With & 42.3 & $39.4-45.2$ & & 264.4 & $257.4-271.4$ & \\
\hline Without & 38.7 & $37.3-40.0$ & & 273.7 & $257.7-289.6$ & \\
\hline Systemic inflammation & & & 0.309 & & & $<0.001$ \\
\hline Four or more markers above median & 38.5 & $36.5-40.5$ & & 284.7 & $274.6-294.8$ & \\
\hline Three or fewer markers above median & 39.8 & $38.3-41.3$ & & 254.8 & $245.9-263.6$ & \\
\hline
\end{tabular}


Table 4 Weighted adjusted HRs and 95\% CIs for incident diabetes according to baseline ox-LDL and ICAM-1 modelled in tertiles

\begin{tabular}{|c|c|c|c|c|c|c|c|c|}
\hline \multirow[t]{2}{*}{ Variable } & \multicolumn{2}{|c|}{ Model 1} & \multicolumn{2}{|c|}{ Model 2} & \multicolumn{2}{|c|}{ Model 3} & \multicolumn{2}{|c|}{ Model 4} \\
\hline & HR & $95 \% \mathrm{CI}$ & HR & $95 \% \mathrm{CI}$ & HR & $95 \% \mathrm{CI}$ & HR & $95 \% \mathrm{CI}$ \\
\hline \multicolumn{9}{|l|}{ ox-LDL } \\
\hline 2nd vs 1 st tertile & 1.33 & $0.99-1.79$ & 1.05 & $0.73-1.52$ & 0.93 & $0.62-1.38$ & 0.92 & $0.62-1.38$ \\
\hline 3 rd vs 1 st tertile & 1.68 & $1.25-2.24$ & 1.21 & $0.85-1.71$ & 1.11 & $0.77-1.61$ & 1.12 & $0.77-1.62$ \\
\hline \multicolumn{9}{|l|}{ ICAM-1 } \\
\hline 2nd vs 1 st tertile & 1.36 & $1.01-1.82$ & 1.46 & $1.01-2.11$ & 1.48 & $1.01-2.18$ & 1.49 & $1.02-2.20$ \\
\hline 3rd vs 1 st tertile & 1.91 & $1.45-2.50$ & 1.73 & $1.20-2.48$ & 1.55 & $1.05-2.29$ & 1.50 & $1.02-2.23$ \\
\hline
\end{tabular}

Model 1: adjusted for age, sex, race and centre

Model 2: adjusted for model 1 variables+fasting glucose

Model 3: adjusted for model 2 variables+waist circumference, HDL-C, triacylglycerol and hypertension

Model 4: adjusted for model 3 variables+CRP

\section{Discussion}

In this large, bi-racial, population-based prospective study, baseline plasma ox-LDL levels were significantly higher in subjects who subsequently developed diabetes than in those who did not develop diabetes. These findings extend the results of two previous smaller, cross-sectional studies that reported elevated circulating ox-LDL levels in individuals with impaired fasting glucose [11] and diabetes [10]. We further found that circulating ox-LDL levels were higher in men than women but did not differ significantly between whites and African Americans.

Plasma ox-LDL levels had a strong positive correlation with LDL-C $(R=0.56, p<0.0001)$ and triacylglycerol $(R=0.28, p<0.0001)$ and a significant negative correlation with HDL-C $(R=-0.25, p<0.0001)$. These findings are in general agreement with those of the Risk Factors in Impaired Glucose Tolerance for Atherosclerosis and

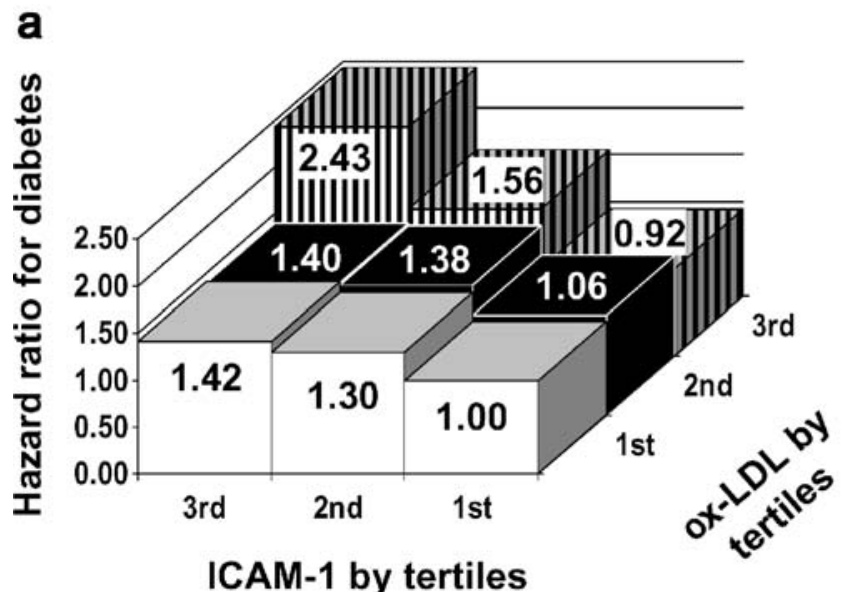

Fig. 1 Adjusted hazard ratios for incident diabetes according to combined baseline ox-LDL and ICAM-1 modelled in tertiles. Compared with individuals with both ox-LDL and ICAM-1 in the lowest tertiles, those with both ox-LDL and ICAM-1 in the highest tertiles had an adjusted HR of 2.43 (95\% CI 1.43-4.13) in model 2 (a),
Diabetes Study [29] and suggest that ox-LDL is primarily associated with dyslipidaemia in individuals in the prediabetic state. Furthermore, individuals with the metabolic syndrome had significantly higher ox-LDL levels than those without the metabolic syndrome ( 42.3 vs $38.7 \mathrm{U} / 1$, $p=0.031$ ), which is in agreement with the Health, Aging, and Body Composition Study in an older population (men and women aged 70-79 years at baseline) [30].

Plasma ox-LDL levels were predictive of incident diabetes in a basic model adjusted for age, sex, race and centre. When estimated in the context of metabolic dysregulation (our fully adjusted models), this association was attenuated and no longer statistically significant. Despite the strong positive correlation of ox-LDL with LDL-C, LDL-C was not predictive of incident diabetes in the basic model (HR=1.24, 95\% CI 0.93-1.64), suggesting that oxidative modification of LDL may be a marker of metabolic changes preceding or accompanying diabetes.

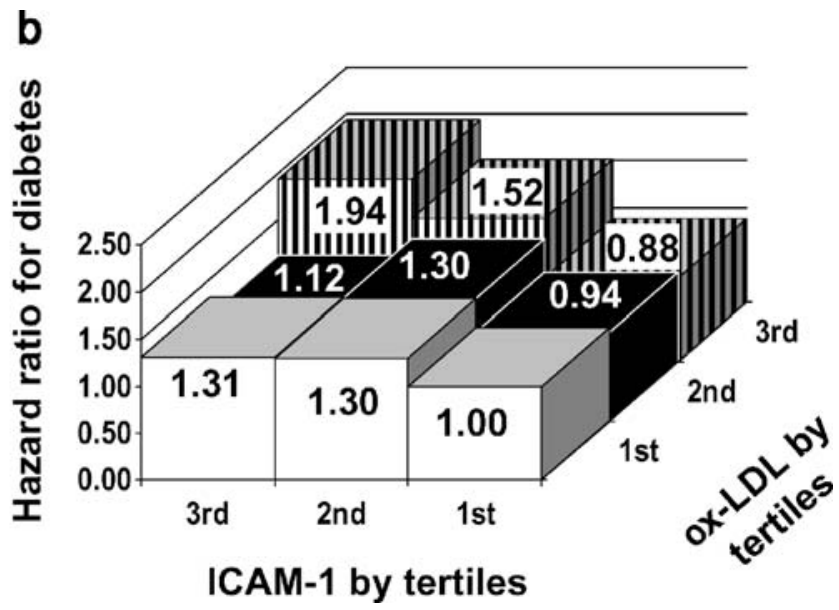

adjusted for age, race, sex, centre and fasting glucose, and $1.94(95 \%$ CI 1.03-3.64) in model 4 (b), adjusted for age, race, sex, centre, fasting glucose, waist circumference, HDL-C, triacylglycerol, hypertension and CRP 
ox-LDL was not significantly correlated with a number of inflammatory markers, including orosomucoid, white blood cell count, IL-6, CRP and fibrinogen, with the exception of a weak correlation with sialic acid. The Atherosclerosis and Insulin Resistance (AIR) Study also showed no correlation between ox-LDL and IL-6, but did show that ox-LDL was positively correlated with CRP and TNF- $\alpha$ [31]. Differences in study populations may account in part for the discrepancy in results; the AIR study enrolled only 58-year-old men of Swedish ancestry, whose cholesterol levels and other cardiovascular risk factors were substantially different from those of the ARIC cohort.

sICAM-1 levels were higher in ARIC subjects who developed diabetes than in those who did not develop diabetes, as well as higher in whites and current smokers than in African Americans and non-smokers, respectively, consistent with our previous report from the ARIC Study that showed an association between incident diabetes and low-grade systemic inflammation in whites but not in African Americans or smokers [12]. Furthermore, sICAM-1 levels correlated positively with a number of inflammatory markers, consistent with previous in vitro and pathological studies showing increased ICAM-1 expression in endothelial cells and other cell types (such as leucocytes and smooth muscle cells) related to vascular inflammation [32-34].

sICAM-1 was independently associated with diabetes in a model adjusted for age, sex, race, centre, metabolic syndrome components and CRP. The Nurses' Health Study also reported that circulating ICAM-1 level was an independent predictor of incident diabetes in women aged $30-55$ years, $94.6 \%$ of whom were white, in similarly adjusted models [19]. sICAM-1 levels were also associated with increased risk of diabetes in middle-aged German men and women in the Monitoring of Trends and Determinants in Cardiovascular Disease/KORA Study [20].

Interestingly, we found a significant interaction between ox-LDL and sICAM-1 in multivariate prediction of incident diabetes. To the best of our knowledge, no other published studies have investigated the interaction between ox-LDL and ICAM-1 in relation to incident diabetes. However, data from animal $[35,36]$ and in vitro $[37,38]$ studies suggest a biological basis for the interaction. Interactions between proinflammatory markers of endothelial dysfunction in the presence of oxidative stress, caused by hyperglycaemia and dyslipidaemia, may modulate diabetes risk in genetically predisposed individuals.

Potential limitations to our study include the use of a single baseline value for ox-LDL and SICAM-1, rather than monitoring fluctuations in levels over time, and the use of fasting plasma samples, which may have underestimated postprandial effects of oxidative stress and inflammation. Also, we found the predictive power of ox-LDL and sICAM-1 for development of diabetes to be modest.
Finally, plasma storage could have led to degradation of sICAM-1 and ox-LDL; however, ox-LDL was previously shown to remain stable in plasma samples stored at $-70^{\circ} \mathrm{C}$ for a short time [39]. Observational prospective studies such as the ARIC Study can assess antecedent factors, although subject to unmeasured or residual confounding, but not causal relationships. However, the information from observational studies such as the ARIC Study does have potential clinical implications for the prevention of diabetes. Measurement of newer markers in addition to traditional factors (glucose, triacylglycerol, BMI, etc) may be useful to identify individuals at increased risk of development of diabetes. More than a century ago, high-dose sodium salicylate was shown to have beneficial effects on diabetes [40], and more recent studies in animals have clearly shown that inhibition of inflammation may have beneficial effects on insulin resistance [41]. Measurement of ICAM-1, ox-LDL or other measures related to inflammation or oxidative stress may be helpful in identifying patient populations in which to test whether novel therapies that inhibit specific pathways related to inflammation or oxidative stress are beneficial in the prevention of diabetes in humans.

Acknowledgements The Atherosclerosis Risk in Communities Study is carried out as a collaborative study supported by National Heart, Lung, and Blood Institute contracts N01-HC-55015, N01-HC55016, N01-HC-55018, N01-HC-55019, N01-HC-55020, N01-HC55021 and N01-HC-55022. Additional funding for this study was provided by National Institute of Diabetes and Digestive and Kidney Diseases Grant RO1-DK56918. The authors thank the staff and participants of the ARIC Study for their important contributions. The atherosclerosis laboratory is supported by donations from $\mathrm{G}$. and C. Mitchell, N. Fares and J. Hines.

Duality of interest The authors have no potential conflicts of interest to declare.

\section{References}

1. Laakso M, Kuusisto J (1996) Epidemiological evidence for the association of hyperglycaemia and atherosclerotic vascular disease in non-insulin-dependent diabetes mellitus. Ann Med 28:415-418

2. DECODE Study Group on behalf of the European Diabetes Epidemiology Group (1999) Glucose tolerance and mortality: comparison of WHO and American Diabetes Association diagnostic criteria. Lancet 354:617-621

3. Kirstein M, Brett J, Radoff S, Ogawa S, Stern D, Vlassara H (1990) Advanced protein glycosylation induces transendothelial human monocyte chemotaxis and secretion of platelet-derived growth factor: role in vascular disease of diabetes and aging. Proc Natl Acad Sci USA 87:9010-9014

4. Rudich A, Tirosh A, Potashnik R, Hemi R, Kanety H, Bashan N (1998) Prolonged oxidative stress impairs insulin-induced GLUT4 translocation in 3T3-L1 adipocytes. Diabetes 47:1562-1569

5. Dandona P, Aljada A, Chaudhuri A, Mohanty P, Garg R (2005) Metabolic syndrome: a comprehensive perspective based on interactions between obesity, diabetes, and inflammation. Circulation111:1448-1454 
6. Ceriello A, Quagliaro L, Piconi L et al (2004) Effect of postprandial hypertriglyceridemia and hyperglycemia on circulating adhesion molecules and oxidative stress generation and the possible role of simvastatin treatment. Diabetes 53:701-710

7. Podrez EA, Schmitt D, Hoff HF, Hazen SL (1999) Myeloperoxidase-generated reactive nitrogen species convert LDL into an atherogenic form in vitro. J Clin Invest 103:1547-1560

8. Steinberg D (1997) Low density lipoprotein oxidation and its pathobiological significance. J Biol Chem 272:20963-20966

9. Holvoet P, Mertens A, Verhamme P et al (2001) Circulating oxidized LDL is a useful marker for identifying patients with coronary artery disease. Arterioscler Thromb Vasc Biol 21:844 848

10. Toshima S, Hasegawa A, Kurabayashi M et al (2000) Circulating oxidized low density lipoprotein levels: a biochemical risk marker for coronary heart disease. Arterioscler Thromb Vasc Biol 20:2243-2247

11. Kopprasch S, Pietzsch J, Kuhlisch E et al (2002) In vivo evidence for increased oxidation of circulating LDL in impaired glucose tolerance. Diabetes 51:3102-3106

12. Duncan BB, Schmidt MI, Pankow JS et al (2003) Low-grade systemic inflammation and the development of type 2 diabetes: the Atherosclerosis Risk in Communities study. Diabetes 52:1799-1805

13. Huo Y, Ley K (2001) Adhesion molecules and atherogenesis. Acta Physiol Scand 173:35-43

14. Hwang S-J, Ballantyne CM, Sharrett AR et al (1997) Circulating adhesion molecules VCAM-1, ICAM-1, and E-selectin in carotid atherosclerosis and incident coronary heart disease cases: the Atherosclerosis Risk in Communities (ARIC) study. Circulation 96:4219-4225

15. Blankenberg S, Rupprecht HJ, Bickel C et al (2001) Circulating cell adhesion molecules and death in patients with coronary artery disease. Circulation 104:1336-1342

16. Ridker PM, Hennekens CH, Roitman-Johnson B, Stampfer MJ, Allen J (1998) Plasma concentration of soluble intercellular adhesion molecule 1 and risks of future myocardial infarction in apparently healthy men. Lancet 351:88-92

17. Leinonen ES, Hiukka A, Hurt-Camejo E et al (2004) Low-grade inflammation, endothelial activation and carotid intima-media thickness in type 2 diabetes. J Intern Med 256:119-127

18. Bluher M, Unger R, Rassoul F, Richter V, Paschke R (2002) Relation between glycaemic control, hyperinsulinaemia and plasma concentrations of soluble adhesion molecules in patients with impaired glucose tolerance or type II diabetes. Diabetologia 45:210-216

19. Meigs JB, Hu FB, Rifai N, Manson JE (2004) Biomarkers of endothelial dysfunction and risk of type 2 diabetes mellitus. JAMA 291:1978-1986

20. Thorand B, Baumert J, Chambless L et al (2006) Elevated markers of endothelial dysfunction predict type 2 diabetes mellitus in middle-aged men and women from the general population. Arterioscler Thromb Vasc Biol 26:398-405

21. Cominacini L, Garbin U, Pasini AF et al (1997) Antioxidants inhibit the expression of intercellular cell adhesion molecule-1 and vascular cell adhesion molecule-1 induced by oxidized LDL on human umbilical vein endothelial cells. Free Radic Biol Med 22:117-127

22. Stewart BW, Nagarajan S (2006) Recombinant CD36 inhibits oxLDL-induced ICAM-1-dependent monocyte adhesion. Mol Immunol 43:255-267

23. ARIC Investigators (1989) The Atherosclerosis Risk in Communities (ARIC) Study: design and objectives. Am J Epidemiol 129:687-702
24. Schmidt MI, Duncan BB, Sharrett AR et al (1999) Markers of inflammation and prediction of diabetes mellitus in adults (Atherosclerosis Risk in Communities study): a cohort study. Lancet 353:1649-1652

25. Expert Panel on Detection, Evaluation, and Treatment of High Blood Cholesterol in Adults (2001) Executive summary of the third report of the National Cholesterol Education Program (NCEP) Expert Panel on Detection, Evaluation, and Treatment of High Blood Cholesterol in Adults (Adult Treatment Panel III). JAMA 285:2486-2497

26. Lin DY (2000) On fitting Cox's proportional hazards models to survey data. Biometrika 87:37-47

27. SAS Institute (1999) SAS/STAT user's guide: version 8. SAS Institute, Cary, $\mathrm{NC}$

28. Shah B, Barnwell B, Bieler G (1997) SUDAAN user's manual. Release 7.5. Research Triangle Institute, Research Triangle Park, $\mathrm{NC}$

29. Hanefeld M, Koehler C, Fuecker K, Henkel E, Schaper F, Temelkova-Kurktschiev $\mathrm{T}$ (2003) Insulin secretion and insulin sensitivity pattern is different in isolated impaired glucose tolerance and impaired fasting glucose: the Risk Factor in Impaired Glucose Tolerance for Atherosclerosis and Diabetes study. Diabetes Care 26:868-874

30. Holvoet P, Kritchevsky SB, Tracy RP et al (2004) The metabolic syndrome, circulating oxidized LDL, and risk of myocardial infarction in well-functioning elderly people in the Health, Aging, and Body Composition cohort. Diabetes 53:1068-1073

31. Hulthe J, Fagerberg B (2002) Circulating oxidized LDL is associated with subclinical atherosclerosis development and inflammatory cytokines (AIR Study). Arterioscler Thromb Vasc Biol 22:1162-1167

32. Gearing AJH, Newman W (1993) Circulating adhesion molecules in disease. Immunol Today 14:506-512

33. Ballantyne CM, Mainolfi EA, Young JB et al (1994) Relationship of increased levels of circulating intercellular adhesion molecule 1 after heart transplantation to rejection: human leukocyte antigen mismatch and survival. J Heart Lung Transplant 13:597-603

34. Nakai K, Itoh C, Kawazoe K et al (1995) Concentration of soluble vascular cell adhesion molecule-1 (VCAM-1) correlated with expression of VCAM-1 mRNA in the human atherosclerotic aorta. Coron Artery Dis 6:497-502

35. Mehrabian M, Allayee H, Wong J et al (2002) Identification of 5-lipoxygenase as a major gene contributing to atherosclerosis susceptibility in mice. Circ Res 91:120-126

36. Shi W, Haberland ME, Jien ML, Shih DM, Lusis AJ (2000) Endothelial responses to oxidized lipoproteins determine genetic susceptibility to atherosclerosis in mice. Circulation 102:7581

37. Sawamura T, Kume N, Aoyama T et al (1997) An endothelial receptor for oxidized low-density lipoprotein. Nature 386:7377

38. Endemann G, Stanton LW, Madden KS, Bryant CM, White RT, Protter AA (1993) CD36 is a receptor for oxidized low density lipoprotein. J Biol Chem 268:11811-11816

39. Pai JK, Curhan GC, Cannuscio CC, Rifai N, Ridker PM, Rimm EB (2002) Stability of novel plasma markers associated with cardiovascular disease: processing within $36 \mathrm{~h}$ of specimen collection. Clin Chem 48:1781-1784

40. Ebstein W (1876) Zur therapie des Diabetes mellitus, insbesondere über die Anwendung des salicylsauren Natron bei demselben. Berl Klin Wochenschr 13:337-340

41. Shoelson SE, Lee J, Goldfine AB (2006) Inflammation and insulin resistance. J Clin Invest 116:1793-1801 